\title{
A comparison of generalization functions and frame of reference effects in different training paradigms
}

\author{
DAVID R. THOMAS, MELISSA LUSKY, and SPENCER MORRISON \\ University of Colorado, Boulder, Colorado
}

\begin{abstract}
Six experiments were carried out to compare go/no-go and choice paradigms for studying the effects of intradimensional discrimination training on subsequent measures of stimulus generalization in human subjects. Specifically, the purpose was to compare the two paradigms as means of investigating generalization gradient forms and frame of reference effects. In Experiment 1, the stimulus dimension was visual intensity (brightness); in Experiment 2, it was line orientation (line-angle stimuli). After learning to respond (or to respond "right") to stimulus value (SV) 4 and not to respond (or to respond "left") to SV2 (in Experiment 1) or SV1 (in Experiment 2), the subjects were tested for generalization (recognition) with an asymmetrical set of values ranging from SV1 to SV11. Go/no-go training produced peaked gradients, whereas choice training produced sigmoid gradients. The asymmetrical testing resulted in a gradual shift of the peak of responding (go/no-go group) or in the point of subjective indifference (PSI; choice group) toward the central value of the test series; thus, both paradigms revealed a frame of reference effect. The results were comparable for the quantitative (intensity) and the qualitative (line-angle) stim. ulus dimensions. Experiment 3 compared the go/no-go procedure with a yes/no procedure in which subjects responded "right" to SV4 and "left" to all other intensities and found no differences between these procedures. Thus the difference in gradient forms in go/no as opposed to (tradi. tional) choice paradigms depends on whether one or two target stimuli are used in training. In Experiment 4, in which visual intensity was used, the shift in the PSI following choice training varied positively with the range of asymmetrical test stimuli employed. In Experiment 5, also with visual intensity, the magnitude of the peak shift following go/no-go training varied as a function of overrepresenting a high or a low stimulus value during generalization testing. Ex. periment 6, with line angles, showed that the PSI following choice training varies in a similar way. The frame of reference effects obtained in these experiments are consistent with an adaptation-level model.
\end{abstract}

Two different paradigms have commonly been used with both animal and human subjects to study the effects of successive intradimensional discrimination training on subsequent measures of stimulus generalization. The procedurally simpler of these is go/no-go training, in which subjects learn to respond to one stimulus-in the case of animals, for appetitive reinforcement-while learning to withhold responding to an alternative stimulus on the same dimension, which, in research with animal subjects, signals extinction. The other procedure requires a choice response, as when subjects are trained to perform one response in the presence of one stimulus and another response in the presence of a different stimulus. With both procedures, training is followed by generalization testing, in which a wide range of stimuli is employed, with reinforcement usually discontinued (in the case of animal subjects) or with feedback discontinued (in the case of humans).

We wish to thank Kelly Mood, Eric Wiertelak, Lorraine Sutton, and Elizabeth Yohn for assistance in the running of subjects. Correspondence concerning this article should be addressed to David $\mathbf{R}$. Thomas, Department of Psychology, Campus Box 345, Boulder, CO 80309-0345.
Historically, investigations of the effects of discrimination training on stimulus generalization have tested predictions of Spence's (1937) theory of discrimination learning in animals, in contrast with predictions based on the more cognitive conception of Köhler (1939) and Krechevsky (1938). We will show in this article that the form of the gradient obtained, which might be thought to favor one theory over the other, depends instead on the training procedure employed.

In recent years, both go/no-go and choice training paradigms have been accompanied by asymmetrical generalization testing (i.e., with more test stimuli to one side of the training values than to the other) in order to investigate psychophysical context effects. In the present experiments, we tested the proposition that such effects (in the two paradigms) have the same underlying cause and found that they vary in a parallel fashion with a common set of procedural manipulations using the same sets of stimuli. As far as we know, this has not been done previously.

In most animal studies, pigeons have been employed in the go/no-go procedure first used by Hanson (1959), who demonstrated the peak shift in postdiscrimination generalization gradients along the wavelength (color) dimension. This phenomenon, maximal responding to a stimu- 
lus value removed from $S+$ in the direction away from $\mathrm{S}-$, was subsequently replicated with human subjects by Doll and Thomas (1967) and others, and it is commonly interpreted as évidence for the summation of excitatory and inhibitory generalization gradients (see Spence, 1937)-although alternative interpretations have been offered, particularly in research with human subjects (see Thomas, 1974). It is important to note that although Spence's theory was originally intended to apply only to nonhuman animals, it has been invoked to explain results obtained with adult humans as well (see, e.g., Baron, 1973; Galizio \& Baron, 1979).

In 1970, Heinemann and Chase, using a choice response paradigm, reported a very different form of postdiscrimination gradient, a sigmoid function rather than a peaked one. When their pigeon subjects learned to respond, say, to the left key in a Skinner box in the presence of a soft noise and to the right key in the presence of a louder one, the birds responded virtually exclusively to the left key in the presence of still softer values and to the right key in the presence of still louder ones. In a parallel experiment, using a go/no-go procedure with the same stimuli, they obtained a similar sigmoid function of stimulus intensity. Heinemann and Chase (1970) therefore suggested that quantitative stimulus dimensions (such as loudness, brightness, etc.) yield generalization gradients of a different form than do qualitative dimensions, such as wavelength of light, line angle, etc. Ernst, Engberg, and Thomas (1971) showed, however, that the result reported by Heinemann and Chase for the go/no-go procedure was probably an artifact of the use of too narrow a range of generalization test values. These investigators used the dimension of light intensity and found that when intensities sufficiently distant from $\mathbf{S}+$ were employed, the gradients obtained from their pigeons, following go/no-go training, had the same peaked form as do gradients along qualitative stimulus dimensions. They suggested that the critical factor determining the form of postdiscrimination gradients is not the type of stimulus dimension employed but rather the (choice vs. go/no-go) paradigm that is used.

Research with human subjects would appear to support the same conclusion. Using the go/no-go paradigm, Doll and Thomas (1967) reported peaked gradients with wavelength stimuli, Baron (1973) reported peaked gradients with tonal frequencies, and Thomas, Svinicki, and Vogt (1973) obtained peaked gradients with the light intensity (brightness) dimension. On the other hand, using the choice paradigm, Johnson (1949a, 1949b) and Sarris and Zoeke (1985) obtained sigmoid gradients with the dimensions of tonal frequency and lifted weights, respectively.

The form of the generalization gradient following discrimination training is of theoretical interest because of its possible implications for the nature of discrimination learning itself. Peaked gradients are consistent with Spence's (1937) view that excitatory and inhibitory tendencies are associated with the (absolute) values of the training stimuli. On the other hand, sigmoid functions are more compatible with the view of Köhler (1939) and
Krechevsky (1938) and others that the subjects learn the ordered relationship between the training values. Given the potential theoretical significance of the difference in gradient forms, it is surprising that there is not a single report of an experiment in which both gradient forms were obtained from the same set of training and test stimuli. Several such experiments with human subjects are reported in the present article. In some, we used a quantitative stimulus dimension, intensity of light, and in others, we used a qualitative stimulus dimension, line angle. It was anticipated that the results obtained with quantitative and qualitative dimensions would be comparable.

A second, more important purpose of the present experiments was to compare go/no-go and choice paradigms as means of investigating frame of reference effects in judgment. Frame of reference or psychophysical context effects are demonstrated when responses to a stimulus differ, depending on its context. Such frame of reference effects have traditionally been investigated in absolute judgment (stimulus rating) tasks but they may be observed in recognition (generalization) tasks with humans and with animals (see Thomas, 1974; Zoeke, Sarris, \& Hofer, 1988).

One type of frame of reference effect has been called "anchoring" by Zoeke et al. (1988). Anchoring is seen when subjects (generally humans) are trained to respond to a particular stimulus and then are tested for recognition (generalization) with an asymmetrical set of test values. They typically show a "central tendency shift," selecting as the original a stimulus closer to the average of all test stimuli. Thomas and Jones (1962) interpreted this effect as indicating that stimuli are encoded in relation to the adaptation level (AL; see Helson, 1964), which is initially set at the value of the training stimulus but shifts during asymmetrical testing as test stimuli are averaged in with the training value to form a constantly changing "prevailing AL."

Thomas et al. (1973) suggested that the peak shift found in postdiscrimination generalization gradients of human subjects following go/no-go training can be accounted for by AL principles. Suppose that a subject were trained to respond to one brightness value, SV (stimulus value) 5 , in a series of nine equally spaced values, and not to respond to $\mathrm{SV} 3$, a dimmer value. The $\mathrm{AL}$ established by this training should fall approximately at SV4, midway between the training values, and the subject should have learned the rule: "respond to AL + 1 unit." During generalization testing with all nine test values, the $\mathrm{AL}$ will shift from SV4 to a higher value such as SV5, the midpoint of the test series. The application of the $A L+1$ unit rule would produce a peak shifted from SV5 to SV6. ${ }^{1}$

Normally, as in the example just given, the training values are near the center of the test series, and thus the change in AL from training to test is small. The shift in $\mathrm{AL}$ and presumably in the location of the peak can be enhanced, however, by the use of training values far removed from the center of the test series, as was done in the present experiments. 
An alternative (choice) discrimination paradigm for studying "anchoring" effects is to train two different responses to two stimuli varying along a dimension and then to test with a range of stimuli asymmetrically spaced around the training values. With this choice paradigm, originally used by Johnson $(1949 a, 1949 b)$ and more recently used by Zoeke, Sarris, and their colleagues (Sarris \& Zoeke, 1985; Zoeke et al., 1988), the effect of the asymmetrical test series is a shift in the point of subjective indifference (PSI), that stimulus value to which both responses are equally likely, from a point approximately midway between the training values to a point nearer the central value of the test series. This result is consistent with the view that the training values are encoded with reference to their relationship to $\mathrm{AL}$, and changes in the PSI during testing track changes in the value of prevailing AL. Thus, the use of training values far removed from the center of the test series should increase the magnitude of the observed shift in the PSI.

Although anchoring effects have been observed with both go/no-go and choice paradigms, no one has directly compared these effects by using the same set of training and test stimuli. We contend that peak shifts and shifts in the PSI with asymmetrical testing both result from the same underlying cause, a shift in Prevailing AL. If our contention is correct, the two paradigms should show similar changes in response tendencies as generalization testing proceeds. Furthermore, manipulations that affect the value of the prevailing $A L$ should have parallel effects in the two paradigms. This hypothesis was tested in the present set of experiments.

It is appropriate to point out that AL theory is not the only one to predict the anchoring effects investigated in these experiments. As will be seen, at least some of the results are equally consistent with Parducci's (1965) range-frequency theory (see Parducci \& Haugen, 1967). The present experiments were not designed to test competing interpretations of psychophysical context effects but rather to seek parallels in the effects obtained with the two training paradigms. The experiments are described from the standpoint of AL theory in order to provide historical continuity with prior papers from this laboratory. Alternative theoretical perspectives will be addressed in the discussion.

\section{EXPERIMENT 1}

The purpose of Experiment 1 was to compare go/nogo and choice paradigms, using the quantitative stimulus dimension of light intensity (brightness). It was predicted that the go/no-go group would yield a generalization gradient that was peaked in form, whereas the choice group would yield a sigmoid gradient. It was further predicted that both groups' gradients would demonstrate an anchoring effect that developed gradually during the course of asymmetrical generalization testing.

\section{Method}

Subjects. The subjects were $\mathbf{4 0}$ students enrolled in introductory psychology classes at the University of Colorado. Approximately two thirds of the the subjects were female, and this proportion of female to male subjects was maintained in the two groups.

Apparatus. Each subject was seated $60 \mathrm{~cm}$ in front of a $60 \times$ $60 \mathrm{~cm}$ panel that was covered with black felt cloth. Approximately at eye level, a $2.7-\mathrm{cm}$-diam aperture was present. The subjects viewed a disk of white light projected onto a translucent glass screen behind the aperture. The light source consisted of a light discrimination apparatus manufactured by the Lafayette Instrument Company (Model 14011), with a 60-W Sylvania clear Decor Lite (60CA9C/BL). Eleven different light intensities were selected so as to be $.188 \log$ units apart. The intensity values and their experimental designations were: SV1, $0.83 \mathrm{fL} ; \mathrm{SV} 2,1.28 \mathrm{fL}$; SV3, $1.97 \mathrm{fL}$; SV4, 3.04 fL; SV5, $4.69 \mathrm{fL}$; SV6, $7.23 \mathrm{fL}$; SV7, $11.2 \mathrm{fL}$; SV8, $17.2 \mathrm{fL}$; SV9, $26.5 \mathrm{fL}$; SV10, $40.9 \mathrm{fL} ; \mathrm{SV} 11,63.0 \mathrm{fL}$. The experiment was conducted in a small, dimly illuminated room. The light reflected from the disk when it was not illuminated was approximately $.01 \mathrm{fL}$. Two push buttons were within reach of the subject's hands, one 2 in. to the right of the other. A response to each button illuminated a light on the experimenter's side of the panel, indicating the response that was made.

Procedure. The subjects were unsystematically assigned to the go/no-go and the choice groups, with 20 subjects in each. After the subject was seated in front of the stimulus panel, the following instructions were read to members of the go/no-go group:

\begin{abstract}
This is an experiment in brightness perception. A light will be presented repeatedly through a small hole in the screen in front of you. Each time it will be presented for 1 sec and may have a different brightness. The first brightness is called the test brightness. Try to remember this brightness because you will have to distinguish if from all the other brightnesses. When you do recognize the test brightness, press the right-hand button. If a subsequent brightness is different from the test brightness, do not press the button. Remember, each time the light is presented it will stay on for only $1 \mathrm{sec}$, so try to respond while the light is on. Indeed, try to respond as quickly yet as accurately as you can. I will tell you whether you are correct on the first few trials; then you will continue without further help. The first light is the test brightness. Keep its brightness in mind. For every light after that, respond by pressing the button as quickly as you can if and only if it is the same as the original light. Any questions?
\end{abstract}

Following any needed clarification of instructions, each subject in the go/no-go group was shown the SV4 stimulus. On the next training trial, SV2 was presented. For this group, training continued for 24 trials with SV4 and SV2 presented 12 times each in unsystematic order. After each correct response, the experimenter said "correct," and after each incorrect response, the experimenter said either "no, that was different from the original" or "no, that was the same as the original," depending on the error. Next, with no interruption in the procecture, the subjects were shown six series of all 11 stimuli; feedback was no longer given. The stimuli were randomized within each series, and the interstimulus interval ranged unsystematically from 2 to $3 \mathrm{sec}$ during both discrimination training and generalization testing.

After subjects in the choice group were seated in front of the stimulus panel, the following instructions were read to them:

\footnotetext{
This is an experiment in brightness perception. A light will be presented repeatedly through a small hole in the screen in front of you. Each time it will be presented for $1 \mathrm{sec}$ and may have a different brightness. For each brightness that you see, you are to respond by pressing either the right-hand or the left-hand button. Your task is to leam the correct response for each brightness. Try to respond while the light is on. Indeed, try to respond as quickly yet as accurately as you can. I will tell you whether you are correct on the first few trials; then you will continue without further help. Any questions?
} 
Only questions dealing with the procedure were answered by the experimenter. Following any needed clarification of instructions, SV4 was shown on the first trial and SV2 was shown on the second trial. Training was continued for 24 trials, with each of these two stimuli presented 12 times in unsystematic order. For all subjects in the group, the correct response for SV2 was on the left button and that for SV4 was on the right. After each correct response, the experimenter said "correct," and after each incorrect response, the experimenter said either "no, that was "right"" or "no, that was 'left,"' depending on the error. Next, with no interruption in the procedure, the subjects were shown six series of all 11 stimuli; feedback was no longer given. The stimuli were randomized within each series, and the interstimulus interval in testing, as during discrimination training, ranged unsystematically from 2 to $3 \mathrm{sec}$.

Subjects in both groups who made more than two errors in the last eight training trials received eight additional training trials before the start of generalization testing. Those who made more than two errors during these additional trials were eliminated from the experiment and replaced.

\section{Results and Discussion}

Only 3 subjects in the go/no-go group and 4 subjects in the choice group required the additional trials, and only 1 in each group needed to be replaced. Subjects given additional training performed no differently from others in their group during generalization testing.

For subjects in the go/no-go group, the mean number of responses to each of the 11 test stimuli is presented in Figure 1. This group shows a decremental gradient with a peak displaced from S+ (SV4) in the direction opposite S- (i.e., it was at SV7), with responding decreasing to more extreme stimulus values.

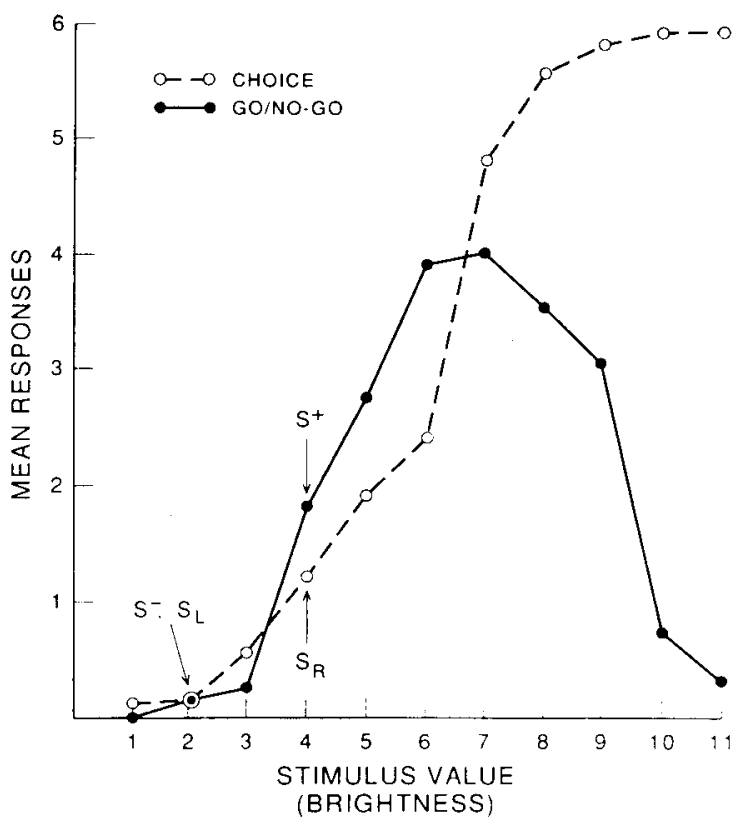

Figure 1. Group mean generalization gradients of the two groups in Experiment 1, based on number of responses (go/no-go group) or number of "right" responses (choice group). The 11 stimulus values, SV1-SV11, refer to the following intensities: SV1, $0.83 \mathrm{fL}$; SV2, 1.28 fL; SV3, 1.97 fl; SV4, 3.04 fL; SV5, 4.69 fl; SV6, $7.23 \mathrm{fL}$; SV7, $11.2 \mathrm{fL}$; SV8, $17.2 \mathrm{fL}$; SV9, $26.5 \mathrm{fL}$; SV10, $40.9 \mathrm{fL}$; SV11, 63.0 fL.
For subjects in the choice group, the mean number of responses made to the "right" button for each of the 11 test stimuli is also presented in Figure 1. As may be seen, the gradient of this group is sigmoidal in form, with the tendency to respond to the right button increasing monotonically with increasing light intensities. The PSI has shifted to a value in excess of SV6.

When peak shift is observed following go/no-go discrimination training in animals, it is typically interpreted in terms of the summation of excitatory and inhibitory gradients as proposed by Spence (1937). Thomas (1974), on the other hand, has accounted for peak shift (in experiments with human subjects) in terms of a change in AL from training to test. A shift in AL from its training to its test value can also explain the location of the PSI in the generalization curve of the choice group (i.e., the stimulus value that is equally likely to be responded to with the left or the right button). Since there were six presentations of each stimulus during testing, the PSI is that stimulus value to which the subjects would have responded with the right button three times. Note that the PSI falls very near the central value of the test series, SV6. Spence's gradient interaction theory provides no basis for a shift in the PSI, since neither stimulus signals nonreinforcement; thus, there is no inhibitory gradient. The AL theory has greater generality than gradient interaction theory has.

Because the peak shift in the go/no-go group is consistent with the prediction based on Spence's (1937) gradient interaction theory, as well as with AL theory, it is important to distinguish between these two interpretations. Spence's theory considers only training parameters, such as the distance between $\mathrm{S}+$ and $\mathrm{S}-$ training values, and ignores any contribution by the location of those values within the generalization test series. Thus the location of the peak should be stationary throughout testing. The $\mathrm{AL}$ theory, on the other hand, demands that the location of the peak should change throughout testing, because continued exposure to the test values results in continual recalculation of the prevailing $\mathrm{AL}$.

Although the shift in AL may occur early in testing, it may be possible to observe the progression of the shift by comparing responding in the first test series and the last test series. Table 1 presents the generalization gradients of the go/no-go group, based on the first test series and the last (sixth) test series. Since each stimulus is presented once within each test series, the gradient indicates the number of subjects who responded by pushing the right button in the presence of each test stimulus. In accordance with prediction, an increasing tendency to respond to higher intensity values during testing may be seen. The shift is most apparent in the reduction of responding to the positive training value, SV4, from 11 out of 20 subjects in the first series to 4 in the last series, and the corresponding increases in responding to SV8 and SV9 from 4 and 5 subjects, respectively, to 14 and 12 . All three of these shifts were significant (for SV4, $\chi^{2}=$ $5.23, p<.05$; for SV8, $\chi^{2}=10.10, p<.01$; for SV9, $\left.\chi^{2}=5.01, p<.05\right)$. 
Table 1

Generalization Gradients From the First and Sixth Test Series in Experiment 1 (Brightness Stimuli)

\begin{tabular}{ccccccccccccc}
\hline \multicolumn{10}{c}{ Stimulus Value } \\
\cline { 2 - 13 } & 1 & 2 & 3 & 4 & 5 & 6 & 7 & 8 & 9 & 10 & 11 \\
\hline \multicolumn{10}{c}{ Go/No-Go Group } \\
1 & 0 & 0 & 1 & 11 & 13 & 16 & 13 & 4 & 5 & 2 & 0 \\
6 & 0 & 0 & 1 & 4 & 10 & 12 & 13 & 14 & 12 & 3 & 2 \\
& \multicolumn{10}{c}{ Choice Group } \\
1 & 0 & 0 & 6 & 9 & 5 & 9 & 18 & 19 & 17 & 19 & 20 \\
6 & 0 & 0 & 0 & 6 & 5 & 11 & 13 & 18 & 16 & 19 & 20 \\
\hline
\end{tabular}

Because the AL presumably shifts within the first test series, it might be possible to demonstrate a corresponding shift in responding within the first series as well. Toward this end, two different sets of random stimulus presentations were used in this experiment, with 10 subjects (within each group) assigned to each set. In one set, SV7 was the second test stimulus experienced; the first was the training value, SV4. In the second set, SV7 was the eighth stimulus experienced. When SV7 was second, only 3 subjects responded to it, whereas when it was eighth, and the subjects had experienced four test values of even greater intensity, all 10 subjects responded to it. This difference is significant: $\left(\chi^{2}=10.77, \mathrm{p}<.01\right)$.

Because the shift in the location of the peak in the go/nogo group and in the PSI in the choice group presumably reflect the same process-a shifting prevailing $A L-i t$ should also be possible to document the gradual shift in the PSI. Table 1 presents the gradients of the choice group, based on the number of subjects responding on the right button during the first and the sixth test series. The differences between the two series are less apparent with this group, since the subjects respond with the right button to the most intense stimulus values from the start of testing. A shift may be seen in the tendencies to respond with the right button to SV3 and SV4, however, which decreases from 6 and 9 out of 20 subjects, respectively, to 0 and 6 . In this group, the shift that occurs within the first test series is particularly remarkable. For half of the subjects, SV4 was the first test stimulus experienced; for half it was the seventh. When it was the first, 9 of 10 subjects responded with the right button, as they had been trained to do. When it was the seventh, however, no subject responded to it with the right button $\left(\chi^{2}=16.0, p<.01\right)$.

\section{EXPERIMENT 2}

Experiment 2 was a replication of Experiment 1, except that the stimulus dimension employed was a qualitative one, line orientation, rather than a quantitative one, brightness. There were other differences in apparatus and procedure necessitated by this change in stimuli, but they were expected to be inconsequential. In addition to providing for the comparison between qualitative and quantitative stimulus dimensions, Experiment 2 serves the function of demonstrating that the shifts to higher inten- sity stimuli observed in Experiment 1 were not attributable to an effect akin to Hull's (1943) "stimulus intensity dynamism." Thomas et al. (1973) had shown peak shift to a less intense stimulus following go/no-go training in which the $S+$ had a dimmer value than did the $S-$. If the results of Experiment 2 parallel those of Experiment 1, a dynamism type of interpretation would be unequivocally ruled out. It would also undermine the possibility that the shifts in responding observed in Experiment 1 were due to the process of sensory adaptation and would point to the need for a more "central" interpretation.

\section{Method}

Subjects. The subjects were $\mathbf{4 0}$ students enrolled in introductory psychology classes at the University of Colorado. Again, approximately two thirds of each group $(n=20)$ were female and one third were male.

Apparatus. The apparatus was a Scientific Prototype two-field tachistoscope (Model $800 \mathrm{~F}$ ). The stimuli consisted of a black line $3.81 \times .32 \mathrm{~cm}$, in orientations of $70^{\circ}$ (counterclockwise from horizontal), $65^{\circ}, 60^{\circ}, 55^{\circ}, 50^{\circ}, 45^{\circ}, 40^{\circ}, 35^{\circ}, 30^{\circ}, 25^{\circ}$, and $20^{\circ}$. The line was centered in a white circular field $7.62 \mathrm{~cm}$ in diameter. The fixation field contained a black dot at its center. Two push buttons were within reach of the subject's hands; one was 2 in. to the right of the other. A response to each button illuminated a light on the experimenter's side of the apparatus, indicating the response that was made.

Procedure. The subjects were unsystematically assigned to the go/no-go and the choice groups, with 20 subjects in each. After the subject was seated in front of the tachistoscope, instructions were read that were similar to those used in Experiment 1, except that the words "line angle" were substituted for the word "brightness" where appropriate. Training was also similar to that in Experiment 1 , except that the $S+$ (for the go/no-go group) or $S$ "right" (for the choice group) was SV4 $\left(55^{\circ}\right)$, and the S- (or S "left") was SVI $\left(70^{\circ}\right)$. In generalization testing, the subjects were shown six series of 11 stimuli: $70^{\circ}, 65^{\circ}, 60^{\circ}, 55^{\circ}, 50^{\circ}, 45^{\circ}, 40^{\circ}, 35^{\circ}$, $30^{\circ}, 25^{\circ}$, and $20^{\circ}$. Feedback was no longer given. The stimuli were randomized within each series. In testing, as during training, the stimuli were presented for $1 \mathrm{sec}$ and the interstimulus interval ranged unsystematically from 5 to $10 \mathrm{sec}$.

Because pilot work had produced less of a shift in responding than had been obtained with brightnesses, it was decided to have the $S+$ and $S$ - farther apart (relative to the range of generalization test stimuli) than in Experiment 1. Thomas, Mood, Morrison, and Wiertelak (1991) had demonstrated a positive relationship between amount of $S+/ S$ - separation and amount of peak shift with lineangle stimuli. As in Experiment 1, for subjects who made more than two errors in the last eight training trials, eight additional training trials were given before the start of generalization testing. Those who made more than two errors during these additional trials were eliminated from the experiment and replaced. Subjects given additional training performed no differently from others in their group during generalization testing.

\section{Results and Discussion}

Only 2 subjects in the go/no-go group needed extra training, and 1 additional subject needed to be replaced. In the choice group, 3 subjects needed extra training and 1 needed replacement.

For subjects in the go/no-go group, the mean number of responses to each of the 11 test stimuli is presented in Figure 2. This group shows a decremental gradient with 


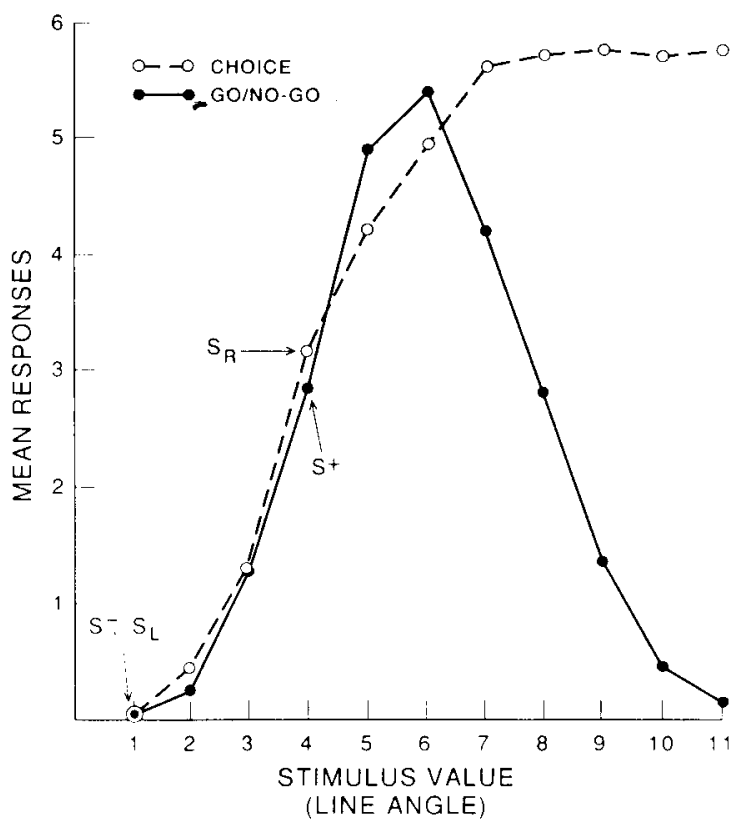

Figure 2. Group mean generalization gradients of the two groups in Experiment 2, based on number of responses (go/no-go group) or number of "right" responses (choice group). The 11 stimulus values, SV1-SV11, refer to the following line angles: SV1, $70^{\circ}$ (counterclockwise from horizontal), SV2, $6^{\circ}$; SV3, $6^{\circ}$; SV4, $55^{\circ}$; SV5, $50^{\circ}$; SV6, $4^{\circ}$; SV7, $40^{\circ}$; SV8, $35^{\circ}$; SV9, $30^{\circ}$; SV10, $2^{\circ}$; SV11, $20^{\circ}$.

a peak displaced from S+ (SV4) in the direction opposite S- (to SV6), with responding decreasing to more extreme stimulus values.

For subjects in the choice group, the mean number of responses made to the right button for each of the 11 test stimuli is also presented in Figure 2. As can be seen, the gradient of this group is sigmoidal in form, with the PSI at a value slightly above SV4 and with the tendency to respond to the right button increasing monotonically with line angles farther removed from SV1. Thus, both gradients are similar in form to their counterparts in Experiment 1.

As in Experiment 1, it was possible to trace shifts in the peak in the go/no-go gradient and in the PSI in the choice gradient during the course of generalization testing. As in Experiment 1, there was evidence of a systematic shift in responding over the course of the test.

Table 2 presents the generalization gradients of the two groups, based on the first test series and the last (sixth) test series. Since each stimulus is presented once in each series, the gradient indicates the number of subjects who responded by pushing the right button in the presence of each test stimulus. In a fashion parallel to the results of Experiment 1, an increasing tendency to respond to values farther removed from SV1 during testing may be seen in the gradients of the go/no-go subjects. The shift is most apparent in the reduction of responding to the positive training value, SV4, from 15 out of 20 subjects in the first series to 7 in the last series, and a corresponding increase in responding to SV8 from 4 subjects to 13 . Both of these shifts were significant (for SV4, $\chi^{2}=6.46, p<.02$; for SV8, $\chi^{2}=8.29, p<.01$ ).

As in Experiment 1, in order to trace changes in responding within the first test series, two different sets of random stimulus presentations were used, with 10 subjects (from each group) assigned to each set. When SV4, the positive training value, was presented first, all 10 subjects responded to it; when it was eighth and the subjects had experienced four more extreme values, only 5 of them responded to it $\left(\chi^{2}=5.40, p<.05\right)$. The shift in responding to SV7 was even more dramatic. When it appeared second, no subject responded to it, whereas when it was tenth, 7 out of 10 subjects did so $\left(\chi^{2}=10.77, p<.01\right)$.

Changes in responding of the choice group that occurred between the first and the sixth test series are less apparent, since the subjects responded with the right button to the stimuli farthest removed from SV1 from the start of testing. They are also less apparent than those which occurred with the brightness stimuli in Experiment 1. Nevertheless, a shift may be seen in the tendency to respond with the right button to SV4 which decreases from 14 out of 20 subjects to 8 . The shift in responding within the first test series was quite dramatic in this group. When SV4 was the first test stimulus experienced, all 10 subjects responded to it, as they had been trained to do, with the right button. On the other hand, when SV4 was the eighth stimulus presented and the subjects had seen four values farther removed from SV1, only 4 of the 10 subjects responded to it with the right button $\left(\chi^{2}=8.57\right.$, $p<.01$ ).

\section{EXPERIMENT 3}

It is clear from the results of the first two experiments that task demands rather than qualitative versus quantitative stimulus dimensions account for the difference between peaked and sigmoidal gradient forms. Yet the task demands of choice and go/no-go paradigms differ in several respects. One is that in the choice paradigm a response is required, whereas in the go/no-go procedure there is room for the subject's bias (i.e., to respond or to withhold responding when in doubt) to influence the results. Experiment 3 was performed to determine whether providing an alternative response in what is otherwise a

Table 2

Generalization Gradients From the First and Sixth Test Series in Experiment 2 (Line-Angle Stimuli)

\begin{tabular}{|c|c|c|c|c|c|c|c|c|c|c|c|}
\hline \multirow[b]{2}{*}{ Series } & \multicolumn{11}{|c|}{ Stimulus Value } \\
\hline & 1 & 2 & 3 & 4 & 5 & 6 & 7 & 8 & 9 & 10 & 11 \\
\hline \multicolumn{12}{|c|}{ Go/No-Go Group } \\
\hline 1 & 0 & 1 & 6 & 15 & 14 & 16 & 7 & 4 & 2 & 1 & 0 \\
\hline 6 & 1 & 0 & 5 & 7 & 18 & 18 & 12 & 13 & 5 & 2 & 1 \\
\hline \multicolumn{12}{|c|}{ Choice Group } \\
\hline 1 & 0 & 0 & 2 & 14 & 13 & 19 & 18 & 19 & 19 & 19 & 19 \\
\hline 6 & 0 & 0 & 2 & 8 & 14 & 16 & 18 & 18 & 19 & 19 & 19 \\
\hline
\end{tabular}


go/no-go paradigm would alter the form and location of the resulting generalization gradient. If subjects are trained to make one response (say, to press a "right' button) in the presence of a target stimulus and another response (say, to press a "left" button) in the presence of all other stimuli, we can separate the effect of having one versus two target stimuli from that of having two choice responses available. Such a procedure has elements of both the traditional go/no-go and choice paradigms and has been called a "yes-no" procedure by van der Heijden and La Heij (1982).

\section{Method}

Subjects. The subjects were $\mathbf{4 0}$ students enrolled in introductory psychology classes at the University of Colorado. Approximately two thirds of each group $(n=20)$ were female and one third were male.

Apparatus. The apparatus was the same as in Experiment 1.

Procedure. The subjects were unsystematically assigned to a go/no-go group and to a yes/no group, with 20 subjects in each. The go/no-go group provided a replication of the comparable group in Experiment 1. The instructions for the yes/no group were altered as follows. Like the go/no-go group, these subjects were told to press the right-hand button if the stimulus presented was the same as the "test stimulus." However, they were told that if the stimulus was different, they were to press the left-hand button. All other aspects of the procedure were identical for the two groups.

\section{Results and Discussion}

Subjects who made more than two errors in the last eight training trials were replaced. There were 2 such subjects in the go/no-go group and 1 in the yes/no group. Subjects given additional training performed no differently from others in their group during generalization testing.

For subjects in the go/no-go group, the mean number of responses to each of the 11 test stimuli is presented in Figure 3. For subjects in the yes/no group, the mean number of responses to the right button is also presented. Both gradients are peaked in form, with the peak shifted from the S+ value SV4 to SV6. There is a slight tendency for the yes/no group to show less shift than the go/no-go group. To test the statistical reliability of this difference, each individual subject's gradient was treated as a grouped frequency distribution, and its mean value was calculated as an index of amount of shift. The mean of these gradient means was 6.13 for the yes/no group compared to 6.63 for the go/no-go group, but the difference was not significant $[t(38)=1.70, p=.10]$. The mean number of responses made by the two groups was also not different $(t<1)$, indicating that the performances of subjects with a bias toward responding when in doubt (in the go/nogo group) were cancelled out by those with a more conservative approach.

\section{EXPERIMENT 4}

The results of Experiments 1 and 2 suggest that the same factor, a shift in prevailing AL from training to test, accounts for the anchoring effects seen with asymmetrical

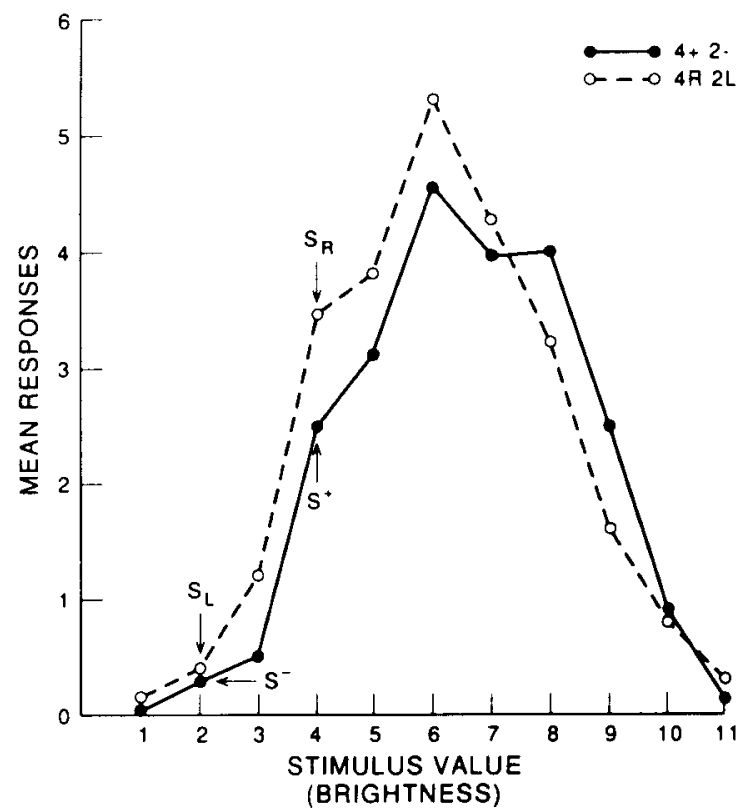

Figure 3. Group mean generalization gradients of the two groups in Experiment 3, based on number of responses (go/no-go group) or number of "right" responses (yes/no) group. See Figure if for stimulus values.

generalization testing in both go/no-go and choice paradigms. In the next three experiments, we tested this conclusion further by manipulating prevailing $\mathrm{AL}$ in two different ways: (1) by varying the range and thus the degree of asymmetry in the generalization test series, and (2) by varying the frequency with which particular generalization test stimuli were presented.

Thomas et al. (1991) recently reported that, with a given set of $S+$ and $S-$ values, the magnitude of the peak shift they obtained varied directly with the range of asymmetrical (line-angle) test values that were used. The wider the range of values (beyond the $S+$ ), the greater would be the shift in prevailing AL during testing and thus the larger the peak shift that would be expected. In Experiment 4 , we used the choice procedure and the brightness dimension, and we predicted that the wider the range of asymmetrical test stimuli employed, the greater would be the shift in the PSI.

\section{Method}

Subjects. The subjects were 40 students enrolled in introductory psychology classes at the University of Colorado. Approximately two thirds of each group $(n=20)$ were female and one third were male.

Apparatus. The apparatus was the same as in Experiment 1.

Procedure. The subjects were unsystematically assigned to widerange and narrow-range groups, with 20 subjects in each. The widerange group was treated identically to, and thus provides a replication of, the choice group of Experiment 1. The subjects were trained to press the right-hand button for SV4 and the left-hand button for SV2, and then they were tested with six random series of 11 test values, ranging from SV1 to SV11. The narrow-range group was 
trained in the same way, but they were tested with seven stimuli, ranging from SV1 to SV7. These stimuli were arranged in nine different random series.

\section{Results and Discussion}

Subjects who made more than two errors in the last eight training trials were replaced. There were 2 such subjects in the wide-range group and 3 in the narrow-range group.

Because of the different numbers of opportunities to respond to the test stimuli in the different groups, the data are reported as the probability of response, averaged for each group (see Figure 4). The difference between the two gradients is quite striking. In each case, the PSI falls slightly above the central test stimulus, which is SV6 in the wide-range group and SV4 in the narrow-range group. To analyze the difference between the two groups' gradients statistically, a PSI was determined for each subject's gradient, on the basis of linear interpolation. The mean PSI of the wide-range group was 6.04, compared to 4.16 in the narrow-range group $[t(38)=6.01, p<$ $.01]$. Thus, the width of the range of asymmetrical test stimuli had the predicted effect on the value of the PSI, just as it did on the amount of peak shift following go/nogo line-angle training in the Thomas et al. (1991) study.

\section{EXPERIMENT 5}

With a given range of test stimuli, one can affect the AL by presenting some stimuli more often than others. If one value is substantially overrepresented, the prevailing AL will shift toward that value and the peak of responding (following single stimulus or go/no-go training)

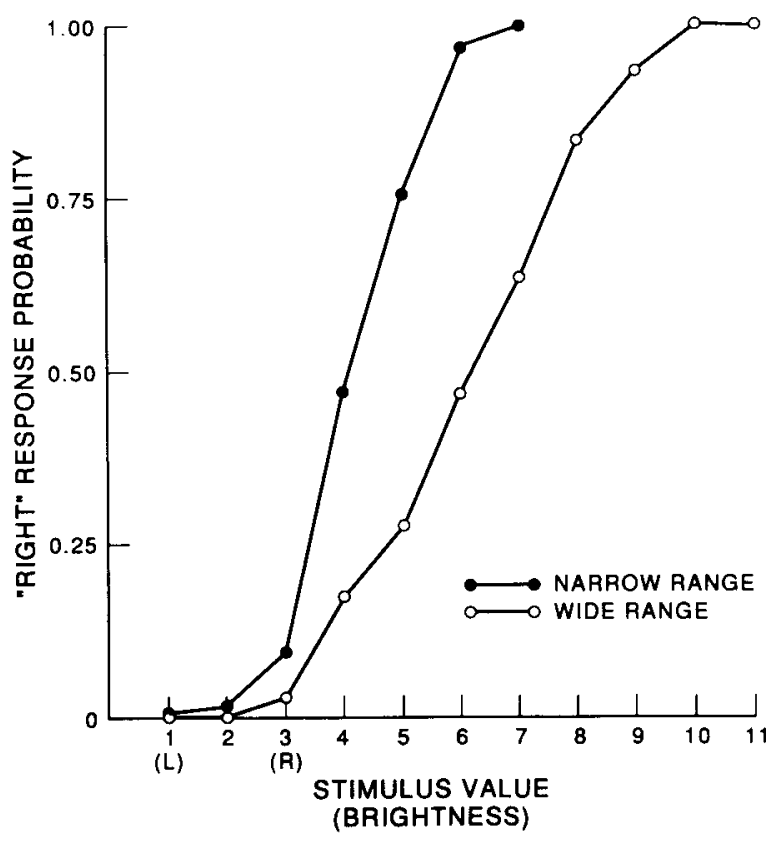

Figure 4. Group mean generalization gradients of wide-range (SV1-11) and narrow-range (SV1 -7) groups in Experiment 4. See Figure 1 for stimulus values. should do likewise. Hebert and Capehart (1969) demonstrated a central tendency shift with lifted weights when the (single) training stimulus was the lightest of five test values, each of which was presented equally often during testing. On the other hand, no shift occurred in a different group of subjects for whom the training value was presented five times as often as any other test value. This finding was subsequently replicated by Hebert, Origlio, and McGuirk (1972) and extended by Hebert, Bullock, Levitt, Woodward, and McGuirk (1974). In the latter study, five different generalization test weights were used, with the training stimulus being the lowest value. In each of five groups, one generalization test stimulus was presented five times as often as the others. The higher the weight value that was overrepresented, the larger the central tendency shift that was obtained.

In Experiment 5, we applied the same manipulation to the measure of peak shift following go/no-go discrimination training, using the light intensity dimension. We predicted that when the $S+$ had a higher value than the $S-$, overrepresenting a high stimulus value during testing would increase the magnitude of the peak shift obtained, whereas overrepresenting a low stimulus value would reduce it.

\section{Method}

Subjects. The subjects were $\mathbf{4 0}$ students enrolled in introductory psychology classes at the University of Colorado. Approximately two thirds of each group $(n=20)$ were female and one third were male.

Apparatus. The apparatus was the same as in Experiment 1.

Procedure. The subjects were unsystematically assigned to two groups, Overrepresent SV2 and Overrepresent SV10. They were trained identically to subjects in the go/no-go group of Experiment 1, with SV4 as S+ and SV2 as S-. They differed only in testing. Instead of six blocks of the 11 test stimuli, these subjects received three blocks, each of which contained one presentation of 10 of the stimuli randomly interspersed among 11 presentations of the remaining one (SV2 or SV10).

\section{Results and Discussion}

Two subjects from each group were replaced for making more than two errors in the last eight training trials. For each subject in each group, a generalization gradient was obtained, based on the probability of responding to each of the 11 test stimuli. The group mean gradients are presented in Figure 5.

The difference between the two gradients is quite striking. Because SV2 was presented 11 times as often as the other values, the peak shift was essentially eliminated. Because SV10 was presented 11 times as often as the others, the magnitude of the peak shift was enhanced relative to that observed in the Overrepresent SV2 group and in the equal representation group that had been run as part of Experiment 3.

To assess the difference in the amount of shift statistically, the mean of each gradient was used as an index of shift. The group means of these mean values were 5.01 and 8.08 in the Overrepresent SV2 and Overrepresent SV10 groups, respectively $[t(38)=3.12, p<.01]$. 


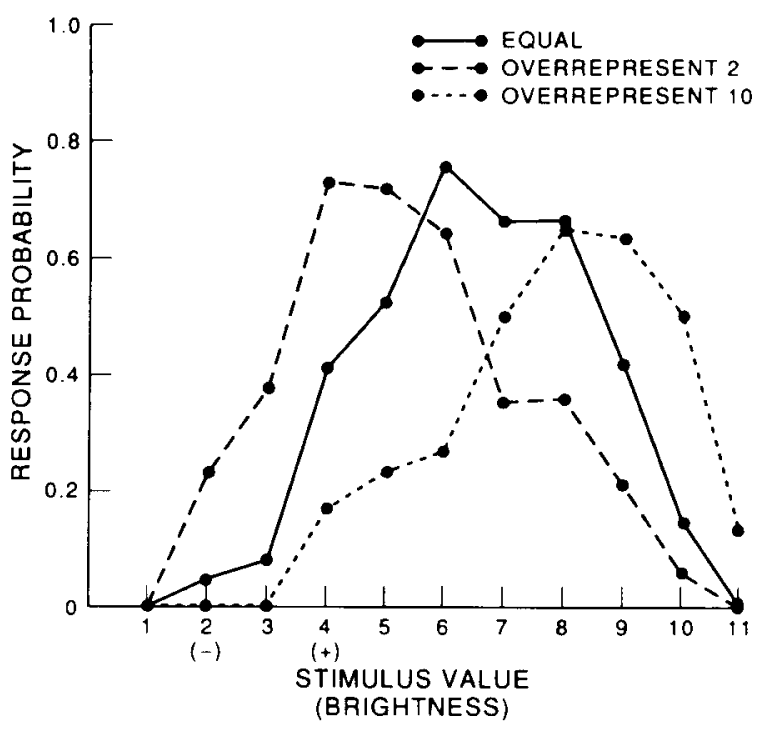

Figure 5. Group mean generalization gradients of Overrepresent SV2 and Overrepresent SV10 groups compared with the gradient of a control (equal representation) group.

Thus, the prediction that overrepresentation during testing would affect amount of peak shift was clearly confirmed.

\section{EXPERIMENT 6}

We designed the final experiment in this series to determine whether the overrepresentation of test stimuli would influence the value of the PSI in the choice paradigm in a manner parallel to its effect on the peak shift following go/no-go training. We tested the hypothesis that it would, with a qualitative stimulus dimension, line angle.

\section{Method}

Subjects. The subjects were $\mathbf{4 0}$ students enrolled in introductory psychology classes at the University of Colorado. Approximately two thirds of each group $(n=20)$ were female and one third were male.

Apparatus. The apparatus was the same as in Experiment 2.

Procedure. The subjects were unsystematically assigned to two groups, Overrepresent SVI and Overrepresent SV7. The instructions and training were similar to those for the choice group in Experiment 2, except that the stimulus to which a left buttonpress was appropriate was SV1 $\left(70^{\circ}\right)$, whereas subjects were trained to press the right button in the presence of SV3 $\left(60^{\circ}\right)$.

In testing, the range of test stimuli from $\mathrm{SV} 1\left(70^{\circ}\right)$ to $\mathrm{SV} 7\left(40^{\circ}\right)$ in $5^{\circ}$ steps was employed. The subjects were exposed to four randomized blocks of test stimuli, within each of which the overrepresented value (SV1 or SV7) was presented six times, whereas the other stimuli were presented once.

\section{Results and Discussion}

Subjects who made more than two errors in the last eight training trials were replaced. There were 2 such subjects in each group. For each subject in each group, a generalization gradient was computed on the basis of the proba- bility of responding with the right button to each of the seven test stimuli. The group mean gradients of the Overrepresent SV1 and Overrepresent SV7 groups are presented in Figure 6.

It is clear from the figure that in accordance with prediction, the Overrepresent SV7 group shows a higher PSI than does the Overrepresent SV1 group. Again, as in Experiment 4 , linear interpolation was used to estimate the PSI for each subject. The mean PSI in the Overrepresent SV7 group was 4.01 , compared to 2.27 in the Overrepresent $S V 1$ group $[t(38)=6.79, p<.01]$.

The magnitude of the effect in Experiment 6 is somewhat smaller than that in Experiment 5. Many factors may account for this difference. In Experiment 5, the overrepresented values were more extreme (i.e., more different from each other) than they were in Experiment 6, and they were overrepresented nearly twice as much (i.e., 11 times the other values, rather than 6 times). The difference between brightness and line-angle stimulus dimensions may also be a factor.

\section{GENERAL DISCUSSION}

The first purpose of this study was to compare the forms of generalization gradients obtained after go/no-go versus choice intradimensional discrimination training. It was shown with both a quantitative stimulus dimension (light intensity) and a qualitative one (line angle) that go/no-go training produces peaked gradients whereas choice training produces sigmoid gradients. This replicates earlier findings in which different stimulus dimensions were used with the two different training paradigms. Thus it seems clear that the form of the obtained generalization gradient

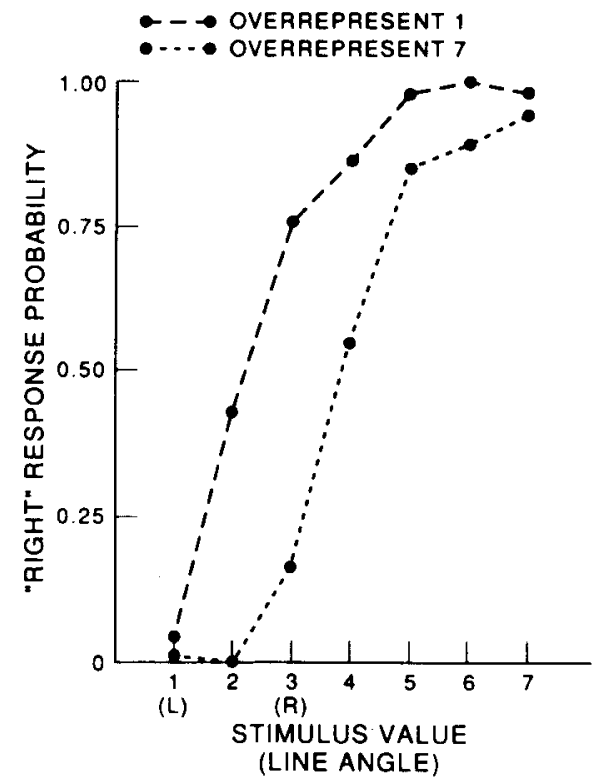

Figure 6. Group mean generalization gradients of Overrepresent SV1 and Overrepresent SV7 groups in Experiment 6. See Figure 2 for stimulus values. 
does not differ according to whether the stimulus dimension is qualitative or quantitative but varies only with the training paradigm employed. Furthermore, the peaked form of the go/no-go gradient is not a result of subjects' having no response available when a test stimulus is perceived as different from the $S+$ training value. The same gradient form (i.e., peaked) results when a response on an alternative button replaces the withholding of the response trained to the S+ (Experiment 3). Thus the critical factor determining the form of the generalization gradient is not whether or not two (symmetrically) different responses are available, as is true in traditional choice but not go/no-go paradigms, but rather whether training involves one target stimulus or two.

As was pointed out in the introduction to this article, the form of the postdiscrimination gradient is of theoretical interest because peaked gradients are more consistent with Spence's (1937) view that subjects acquire response tendencies to the absolute values of the training stimuli, whereas sigmoid gradients are more consistent with a relational approach, such as that proposed by Köhler (1939) and Krechevsky (1938). According to the traditional relational view, the subject solves the discrimination problem relationally by learning to respond to the larger (or smaller), the brighter (or dimmer), etc., of the training stimuli and applies this relational rule during testing. A problem for this simple relational view is that a postdiscrimination generalization gradient following go/nogo training shows a peak of response strength (i.e., a single maximal value), beyond which responding decreases as stimuli still farther from $\mathrm{S}+$ are tested. This decrease is inconsistent with the notion that the training stimuli were encoded relationally.

Although the finding of a sigmoid gradient following choice training is indeed consistent with the traditional relational view, a simpler "absolute" interpretation is available. The (forced) choice procedure requires the subject to respond by pressing either the left or the right button in the presence of every test stimulus. A response of "neither one" is not acceptable. When the trained response to, say, the brighter of two stimuli is to press the right button, a stimulus that is brighter still will necessarily be more similar to the training value associated with a right response than to the training value associated with a left response. The principle of stimulus generalization thus predicts a right response for all brighter stimuli. ${ }^{2}$

Thus the difference in the forms of the gradients obtained following go/no-go and choice training is based on the task demands of the two paradigms and does not reflect any difference between the two in relational versus absolute encoding of the training stimuli. The proof that the training stimuli are encoded relationally lies elsewhere, as, for example, in the shifting of responding that occurs during the course of asymmetrical generalization testing.

It is appropriate to point out that unlike in the go/no-go procedure, in which subjects are instructed to respond to a particular (single) stimulus value, in the choice procedure the instructions are more ambiguous. Subjects are given no indication of how they should respond to test stimuli that they have not experienced during training and they must therefore extrapolate from the training experience to the test situation. Quite possibly, subjects differ in how they interpret the task-for example, they may encode the stimuli relationally, absolutely, or in both ways. It is therefore a testament to the power of the task demands that virtually all subjects produce a sigmoid gradient. Perhaps this should not be surprising, since it occurs also with animal subjects despite various procedural manipulations. Heinemann and Chase (1970) obtained sigmoid gradients from pigeons while testing them without reinforcement, whereas Zoeke et al. (1988) obtained sigmoid gradients in chickens while reinforcing them on every choice trial regardless of which response was made.

This brings us to the major purpose of the present study, which was to compare go/no-go and choice training paradigms as means of investigating " anchoring" effects in judgment. We proposed that the mechanism underlying peak shift, following go/no-go training, and the shift in the PSI, following choice training, were one and the same-that is, a shift in prevailing AL during asymmetrical generalization testing. The evidence presented in support of this interpretation comprised three different sorts. The AL formulation (see Thomas, 1974) predicts that the location of the peak following go/no-go training and of the PSI following choice training will shift gradually during the course of asymmetrical generalization testing as prevailing AL shifts. The gradualness of the shifts was demonstrated in Experiments 1 and 2.

A second factor that should affect prevailing AL during testing is the range of asymmetrical test stimuli employed. Thomas et al. (1991) showed that a wider range results in a larger line-angle peak shift following go/nogo training. Experiment 4 in the present study demonstrated a larger shift in the PSI as a result of the same manipulation with the brightness dimension.

A third factor that should affect prevailing $\mathrm{AL}$ is the overrepresentation of certain (high or low) stimulus values during generalization testing. Experiments 5 and 6, using go/no-go and choice paradigms, respectively, and brightness and line-angle dimensions, respectively, demonstrated greater shifts in the peak or the PSI, respectively, as a high test value was overrepresented, relative to groups with which a low test value was overrepresented.

Although the evidence in favor of an AL interpretation of the effects of asymmetrical generalization testing is substantial, it has its shortcomings. One of these is the difficulty of estimating the prevailing $\mathrm{AL}$ in the absence of actually measuring it. It must be remembered that $A L$ is the psychological average stimulus and that it may differ substantially from the physical average. For example, Thomas et al. (1973) used the rating scale method (Helson, 1964) to empirically determine the AL of a set of nine logarithmically equally spaced intensity values and obtained an AL value of 6.38, which was well above 5.0, the central value of the series. Furthermore, Newlin, Rodgers, Dickson, Strub, and Thomas (1978) determined 
that the AL of a given set of intensity values varied as a function of the subject's "preexperimental" experience in judging other intensities. Thus, the AL established in training is quite probably not midway between the two training values and might not even be between the two of them. This does not contradict the contention that the training stimuli are encoded in relation to $\mathrm{AL}$, but it makes the absolute determination of the value of $\mathrm{X}$ in the $\mathrm{AL}+\mathrm{X}$ formulation impossible. Furthermore, although prevailing $\mathrm{AL}$ results from a combination of the training and testing experience, there is reason to believe that the training experience receives additional weight in the computation; otherwise, the central tendency shift observed after a single exposure of a training stimulus would not occur gradually, as it does (see Thomas, 1974). There is no obvious formula for determining how much weight to give the training experience in the computation of prevailing $\mathrm{AL}$.

A further complication is the fact that the $\mathrm{AL}+\mathrm{X}$ formulation assumed that $X$ would be established by the training experience and would remain constant throughout testing. A recent study by Thomas et al. (1991) has indicated that this assumption is incorrect. These investigators trained their subjects to respond to two different line angles with an S- midway between them, and then tested the subjects with a symmetrical test series covering either a narrow or a wide range beyond the two $S+s$. Both groups showed bimodal gradients, with the peaks displaced from the $S+$ values so as to be farther removed from the single $S-$. Furthermore, the peak shifts were greater when the test range was wider. Since symmetrical generalization testing should not change the $\mathrm{AL}$ from its training value, the fact that the peaks shifted and the difference in the amount of shift in the two groups suggests that the subjects had learned the rule "respond to AL $\pm X$." The size of X then increased with the switch from the training to the generalization test stimuli, and it increased more when the test series had a wider range. This notion that $\mathrm{X}$ varies in proportion to the range of test values is consistent with the basic premise of AL theory that stimuli are always judged relationally rather than absolutely. We may think of the response rule as "respond to $\mathrm{AL} \pm$ a little (or a lot)," but what seems a little (or a lot) should change when the range of values considered increases. The importance of the relativity of $X$ to our present concern is that even if we can empirically determine the value of the prevailing $\mathrm{AL}$, we cannot predict the location of the peak because the size of $X$ will vary with the range of test stimuli employed. Thus, the go/nogo paradigm is highly sensitive to context effects and is useful in demonstrating them, but it is limited to ordinal predictions based on the AL formulation.

The choice paradigm fares somewhat better as a test of $\mathrm{AL}$ effects. Although the problems relating to the determination of prevailing AL still apply, the assumption is that the PSI will fall at the value of prevailing $A L$. This hypothesis is clearly subject to empirical test. Furthermore, the determination of the PSI is generally unambiguous, whereas many go/no-go gradients are relatively flat and are sometimes bi- or trimodal. Presumably, in the choice paradigm the subject learns the rule "respond with the right button for $A L+X$ and with the left button for $\mathrm{AL}-\mathrm{X}$." $A$ change in the size of $\mathrm{X}$ as generalization testing progresses should have no effect on the value of the PSI. Any stimulus perceived as greater in magnitude than the AL (by whatever amount) should result in a "right" response, because it will necessarily be more similar to $A L+X$ than to $A L-X$. Because the value of $X$ influences the location of maximal go/no-go responding but not that of the PSI in choice responding, it makes the former paradigm more sensitive to context effects but less useful as a test of specific quantitative predictions.

As mentioned in the introduction, anchoring effects can be interpreted in alternative ways. The shift in the PSI obtained with the choice procedure is particularly amenable to interpretation according to Parducci's (1965) range-frequency model, which suggests that subjects tend to respond with equal frequency with each response available to them (e.g., with the right or the left button). In order to accomplish this, they divide the stimulus range in such a way that the median stimulus value becomes the PSI. An increase in the range of asymmetrical test stimuli, as in Experiment 4, or a disproportionate presentation of one test stimulus relative to all others, as in Experiment 6, would affect the median stimulus value and thus the PSI in both of these experiments. These manipulations would change the mean in a similar fashion, however, so that $A L$ and range-frequency interpretations are equally appropriate; the data cannot discriminate between them.

Parducci and Haugen (1967) demonstrated that comparative judgments (i.e., greater than or less than a standard) were influenced by the median of the judged stimuli even when the mean was held constant. This effect is inconsistent with AL theory. It does not mean, however, that when the mean stimulus does change this is not the basis for a change in judgment. Rather, it indicates that the anchoring effect may have more than one possible cause.

Our preference for the AL interpretation in this article is based on its broader applicability. Recall that we originally applied the AL model to explain the central tendency shift obtained by following single stimulus training with asymmetrical generalization testing. We subsequently extended the model to explain the peak shift following go/nogo training. In both of these situations, there is no tendency for subjects to identify stimuli as the same as or different from the original (or $S+$ ) with equal frequency; and the proportion of same to different responses does not change during generalization testing-only the stimuli to which those two responses are made change. Unlike the choice paradigm, in which the PSI must shift in order to accommodate the frequency principle, single stimulus or go/no-go procedures do not require a shift in order to accomplish the same objective. It is thus not clear that rangefrequency theory would predict the anchoring effects that we have observed with the use of these other paradigms. 
It seems parsimonious to us to prefer a theoretical perspective that encompasses anchoring effects in all of the paradigms in which they have been demonstrated.

\section{REFERENCES}

Baron, A. (1973). Post discrimination gradients on a tone continuum. Joumal of Experimental Psychology, 101, 337-342.

Doll, T. J., \& Thomas, D. R. (1967). Effects of discrimination training on stimulus generalization in human subjects. Journal of $E x$ perimental Psychology, 75, 508-512.

Ernst, A. J., Engberg, L., a Thomas, D. R. (1971). On the form of stimulus generalization curves for visual intensity. Joumal of the Experimental Analysis of Behavior, 16, 177-180.

Galzio, M., * BARON, A. (1979). Human postdiscrimination gradients: The effects of three-stimulus discrimination training. Animal Learning \& Behavior, 7, 53-56.

HANSON, H. M. (1959). Effects of discrimination training on stimulus generalization. Journal of Experimental Psychology, 58, 321-333.

Hebert, J. A., Bullock, M., LevitT, L., Woodward, K. G., \& McGuirk, F. D., III. (1974). Context and frequency effects in the generalization of a human voluntary response. Joumal of Experimental Psychology, 102, 456-462.

Hebert, J. A., CAPEharT, J. E. (1969). Generalization of a voluntary response as a function of presentation frequency of the training stimulus in testing. Psychonomic Science, 16, 315-316.

Hebert, J. A., Origlio, D. P., \& McGuirk, F. D. (1972). Training and testing effects in the generalization of a voluntary response. Psychonomic Science, 26, 209-210.

Heinemann, E. G., Chase, S. (1970). On the form of stimulus generalization curves for auditory intensity. Joumal of Experimental Psychology, 84, 483-486.

HeLson, H. (1964). Adaptation-level theory. New York: Harper \& Row.

HuLl, C. L. (1943). Principles of behavior. New York: AppletonCentury-Crofts.

Johnson, D. M. (1949a). Generalization of a reference scale for judging pitch. Journal of Experimental Psychology, 39, 316-321.

Johnson, D. M. (1949b). Learning function for a change in the scale of judgment. Joumal of Experimental Psychology, 39, 851-860.

KöHLER, W. (1939). Simple structural functions in the chimpanzee and in the chicken. In W. D. Ellis (Ed.), A source book of Gestalt psychology (pp. 217-227). New York: Harcourt, Brace.

KRECHEVSKY, I. (1938). A study of the continuity of the problem solving process. Psychological Review, 45, 107-133.

Newlin, R. J., Rodgers, J. P., Dickson, J. F., Jr., Strub, H., \& Thomas, D. R. (1978). The central tendency effect in stimulus generalization: Effects of establishing a "preexperimental" frame of reference. Perception \& Psychophysics, 24, 161-167.

PARDUCCI, A. (1965). Category judgment: A range-frequency model. Psychological Review, 72, 407-418.
Parducci, A., \& Haugen, R. (1967). The frequency principle for comparative judgments. Perception \& Psychophysics, 2, 81-82.

SARRIS, V., ZOEKE, B. (1985). Tests of a quantitative frame-ofreference model: Practice effects in psychophysical judgments with different age groups. In G. d'Ydewalle (Ed.), Cognition, information processing, and motivation (pp. 71-78). Amsterdam: NorthHolland.

SPENCE, K. W. (1937). The differential response in animals to stimuli varying within a single dimension. Psychological Review, 44, 430-444.

Thomas, D. R. (1974). The role of adaptation-level in stimulus generalization. In G. W. Bower (Ed.), The psychology of learning and motivation (Vol. 8, pp. 91-145). New York: Academic Press.

Thomas, D. R., JonEs, C. G. (1962). Stimulus generalization as a function of the frame of reference. Journal of Experimental Psychology, 63, 73-80.

Thomas, D. R., Mood, K., Morrison, S., * Wiertelak, E. (1991). Peak shift revisited: A test of alternative interpretations. Journal of Experimental Psychology: Animal Behavior Processes, 17, 130-140.

Thomas, D. R., Svinick, M. D., \& VoGt, J. (1973). Adaptation-level as a factor in human discrimination learning and stimulus generalization. Journal of Experimental Psychology, 97, 210-219.

VAN der Heijden, A. H. C., \& L Hev, W. (1982). The array size function in simple visual search tasks: A comparison between "gono go" and "yes-no" tasks under conditions of high and low targetnoise similarity. Psychological Research, 44, 355-368.

Zoeke, B., SARris, V., Hofer, G. (1988). Psychophysical context effects in chickens (hubbards). International Joumal of Comparative Psychology, 1, 167-178.

\section{NOTES}

1. When the $S+$ is at a higher stimulus value than the $S-$, the generic form of the response rule suggested by Thomas et al. (1973) is "respond to $\mathrm{AL}+\mathrm{X}$," where $\mathrm{X}$ is the distance in stimulus units between the $\mathrm{AL}$ and the $\mathrm{S}+$ value. If the $\mathrm{AL}$ were not at the midpoint between the $\mathrm{S}+$ and the $S-$, the value of $X$ would necessarily be different from the one unit specified in the example given. Without empirically determining the actual value of the $\mathrm{AL}$ in training, it is still possible to make ordinal predictions by using the $A L+X$ formulation. For example, it necessarily follows that a wider spacing between $S+$ and $S-$ will increase the value of $\mathbf{X}$ and result in a greater magnitude of peak shift.

2. This statement must be qualified a bit. If a test stimulus were so far removed from the training values that the subject could not determine which value it was closer to, choice responding should revert to chance. This has not actually occurred in any study of which the present authors are aware.

(Manuscript received October 8, 1991; revision accepted for publication December 27, 1991.) 